\title{
Novedades para la brioflora de la Sierra de Gredos (Sistema Central, España), con especial énfasis en la comunidad de Extremadura
}

\author{
Modesto Luceño1, José Ignacio Márquez-Corro', Samuel Guerra-Cárdenas', Rogelio Sánchez \\ -Villegas ${ }^{1}$, Ismael Jurado-Castillo1, Eva Rodríguez-Velasco', Juan Carlos Rodríguez-Escobar', Manuel \\ Sánchez-Villegas ${ }^{1}$, Carlos Cerrejón ${ }^{2}$, Fernando Estévez-Rodríguez ${ }^{3}$ \& Jesús Muñoz ${ }^{4}$ \\ 1 Universidad Pablo de Olavide, Ctra. de Utrera km 1, E-41013 Sevilla. \\ 'Institut de recherche sur les forêts (IRF). Université du Québec en Abitibi-Témiscamingue, 445 Boul. de l'Université, \\ Rouyn-Noranda, Québec J9X 1C5, Canada. \\ ${ }^{3}$ Travesía de las Eras, 6, E-10612 Jerte, Cáceres \\ ${ }^{4}$ Real Jardín Botánico (RJB-CSIC), Plaza de Murillo 2, E-28014 Madrid.
}

\section{Correspondencia}

M. Luceño

e-mail:mlucgar@upo.es

Recibido: 31 octubre 2017

Aceptado: 27 noviembre 2017

Publicado on-line: diciembre 2017

\begin{abstract}
Resumen
En este artículo se presentan numerosas novedades corológicas de interés, basadas en las colectas llevadas a cabo durante los años 2016 y 2017 en los pisos oro y crioromediteráneo de la sierra de Gredos, zonas que habían sido escasamente colectadas. Entre los resultados destacamos el hallazgo de Douinia ovata, desconocida de la región mediterránea española, y de Sciurohypnum reflexum y Tortula hoppeana, nuevos para el Sistema Central en su conjunto. Destacamos también 4 hepáticas y 24 musgos nuevos para la Comunidad Autónoma de Extremadura, así como otras no conocidas para la sierra de Gredos o las provincias de Ávila o Salamanca. Por último, se incluyen citas de nuevas poblaciones de briófitos muy raros en Gredos o el Sistema Central en su conjunto.
\end{abstract}

Palabras clave: Briófitos, hepáticas, musgos, novedades corológicas.

\begin{abstract}
Novelties of the Sierra de Gredos bryoflora (Central System, Spain), with special remarks on the autonomous community of Extremadura

This study present new records of bryophytes based on the collections made along the years 2016 and 2017 in both oro and cryoromediterranean bioclimatic subzones of Gredos mountain range, which were scarcely collected. Among the findings, we highlight Douinia ovata, unknown up to date for the Mediterranean Region of Spain, and Sciuro-hypnum reflexum and Tortula hoppeana, both new to the Central System. Also, we highlight the finding of 4 liverworts and 24 mosses news to the Autonomous Community of Extremadura, some of which also unknown either for Gredos mountain range or Ávila or Salamanca provinces. Finally, records of new populations of very rare bryophytes in Gredos or the Iberian Central System are included.
\end{abstract}

Key words: Bryophytes, liverworts, mosses, new records.

\section{Introducción}

El presente artículo es la continuación de los estudios acerca de la brioflora de la sierra de Gredos, cuyos primeros resultados fueron recogidos en dos publicaciones previas (Luceño et al., 2016a; Luceño et al., 2017). Dicha sierra contiene las mayores elevaciones del Sistema Central ibérico y está enclavada en la región mediterránea de la Península Ibérica (Luceño et al., 2016b), si bien, 
su diversidad microclimática propicia un mosaico de ecosistemas que han favorecido la instalación de organismos de diferentes requerimientos ecológicos: eurosiberianos, bóreo-alpinos, oceánicos, subtropicales, etc. (véanse Luceño et al. 2016a y 2017 para mayor detalle acerca de las características climáticas, geológicas y biogeográficas de la sierra).

Los datos aquí presentados son el resultado de las prospecciones llevadas a cabo durante los años 2016 y 2017 en valles y crestas poco o nada explorados de los tres macizos que comprenden la cadena principal de la sierra de Gredos (ver Luceño et al., 2017 para más detalles sobre el área estudiada).

\section{Materiales y métodos}

Todos los materiales estudiados han sido colectados por los autores a lo largo de diez campañas llevadas a cabo desde la primavera del año 2016 hasta comienzos del otoño de 2017. Dichos materiales han sido depositados en el herbario UPOS (Universidad Pablo de Olavide). Las novedades están resaltadas con un asterisco $\left({ }^{*}\right)$ delante del taxon en caso de ser novedades regionales, o junto al nombre de la provincia, si se trata de novedades provinciales.

La nomenclatura seguida es la de Ros et al. (2013) para musgos, con excepción de Bryum muehlenbeckii Bruch \& Schimp. (Guerra et al., 2010) y Andreaea heinemannii (Dirkse \& LosadaLima, 2010), y la de Söderström et al. (2016) para hepáticas y antocerotas.

\section{Resultados y discusión}

Destacamos dos novedades para la brioflora del Sistema Central ibérico, otra para el tramo español de dicha cordillera, una para la provincia de Ávila, cinco para la de Salamanca y 28 táxones nuevos para la Comunidad de Extremadura.

\section{HEPÁTICAS}

\section{Diplophyllum taxifolium (Wahlenb.) Dumort.}

${ }^{*}$ Cáceres. Macizo central de Gredos, Tornavacas, cabecera de la garganta de San Martín, 40¹3'47.07'N / 5³7'32.89”W, 1975 m, pedregales, 02-09-2017; I. Jurado-Castillo et al., 90IJC17; UPOS-104309. Idem, circo de la Angostura, 40¹2'29.52'N / 5³7'19.54"W, $2175 \mathrm{~m}$, roquedos y fisuras graníticas orientadas al norte, 23-09-2017; R. Sánchez-Villegas et al., 673RSV17; UPOS-104346.

Especie más común de lo que se suponía hasta ahora en el Sistema Central (véase Luceño et al., 2016a).
${ }^{*}$ Douinia ovata (Dicks.) H.Buch

Ávila. Macizo occidental de Gredos, supra laguna del Duque, 40¹7'50.03"N / 541'33.10'W, 1823 m, repisa rocosa expuestas al norte, 01-10-2016; S. GuerraCárdenas et al., 602SGC16; UPOS-104140.

Especie eurosiberiana de marcado carácter oceánico que en la Península Ibérica era conocida exclusivamente de ciertas áreas del norte (Hespanhol et al., 2010; Casas et al., 1985). Se trata, por tanto, de la primera referencia para el Sistema Central español.

\section{*Gymnomitrion alpinum (Gottsche ex Husn.) Schiffn.}

Ávila. Macizo central de Gredos, Zapardiel de la Ribera, aledaños del pico Plaza de Toros, 40¹6'51.52'N / 5¹9'06.81' 'W, $2186 \mathrm{~m}$, bloques graníticos orientados al norte, 15-10-2016; S. Guerra-Cárdenas \& M. Luceño, 696SGC16; UPOS-104074. Idem, Bohoyo, Sierra Llana, circo de la Cocinilla, 40¹5'19.49'” / 5²5'03.72'”W, 2237 $\mathrm{m}$, fisuras de granito fuertemente innivadas, 05-07-2017; R. Sánchez-Villegas et al., 209RSV17; UPOS-104264.

Este taxon, de apetencias claramente bóreoalpinas (Paton, 1999), ha sido citado [como Marsupella alpina (Gottsche ex Limpr.) H. Bern.] de las cumbres de la sierra de Guadarrama (Casares Gil \& Beltrán Bigorra, 1912), así como de Gredos [puerto del Pico (Soria et al., 1987) y sierra de Béjar (Elías, 1988)] y de la Peña de Francia (Elías, 1988). Hemos conseguido estudiar las muestras recolectadas por Elías (1988) y, en nuestra opinión, pertenecen a Marsupella funckii (F. Weber \& D. Mohr) Dumort. (SALAbryo 825, 826, 827) o Cephaloziella divaricata (Sm.) Schiffn. (SALA-bryo 831). No hemos tenido ocasión de estudiar la muestra citada por Soria et al. (1987) procedente del puerto del Pico; sin embargo, en dicha localidad crece abundante $M$. funckii. Esta última es de apariencia muy similar a G. alpinum, especialmente en lo referente a los caracteres vegetativos. En general, los filidios de Gymnomitrion alpinum muestran lóbulos con frecuencia obtusos y son perceptiblemente envainadores en la base, mientras que en M. funckii los filidios no son envainadores y presentan lóbulos casi siempre agudos. La presencia de inflorescencias femeninas resulta muy clarificadora para distinguir ambas especies, puesto que las de $G$. alpinum muestran un periginio muy corto y el perianto está reducido a escamas cuyas células son más o menos isodiamétricas y tienen las paredes engrosadas; por el contrario, las inflorescencias femeninas de $M$. funckii exhiben periginio y perianto bien desarrollado, con las células apicales del último rectangulares y de paredes finas; además, las brácteas de la inflorescencia femenina están imbricadas en $G$. 
alpinum y más o menos separadas en $M$. funckii (fig. 1). Por añadidura, M. funckii es una hepática de apetencias montanas que en Gredos no supera los $2000 \mathrm{~m}$, salvo raramente en las laderas de la vertiente meridional, mientras que $G$. alpinum habita en zonas de cumbres expuestas al norte y muestra un acusado carácter quionófilo.

\section{*Isopaches bicrenatus (Schmidel ex Hoffm.) H.Buch \\ Ávila. Macizo central de Gredos, Navalguijo,} sendero de la Laguna de los Caballeros, $40^{\circ} 14^{\prime} 50.67^{\prime \prime} \mathrm{N}$ / 5³0'45.17''W, $1241 \mathrm{~m}$, brezal, 21-03-2016; M. Luceño \& S. Guerra-Cárdenas, 30ML16; UPOS-103304.

Novedad para la sierra de Gredos. Esta hepática se distribuye de forma dispersa por la mitad norte de la Península y su localidad más cercana conocida se encuentra en la sierra de Gata (Casas et al., 1988).

\section{Jungermannia exsertifolia subsp. cordifolia (Dumort.) Váňa}

Ávila. Macizo oriental de Gredos, Villarejo del Valle, arroyo Rioseco, 40¹8'21.89' N / 459'27.64'W, 1170 m, sumergida en arroyo, 09-04-2017; S. Guerra-Cárdenas et al., 181SGC17; UPOS-104320. Salamanca. Macizo occidental de Gredos, Candelario, sendero del Travieso a Hoya Moros, 40¹9'08.60"N / 544'09.24'W, 2038 $\mathrm{m}$, granitos en recovecos recorridos por arroyo, 01-092016; S. Guerra-Cárdenas et al., 486SGC16; UPOS103705. Idem, circo de Hoya Mayor, 40²0'06.07'N /
543'18.60'W, 1924 m, lecho de arroyo, 22-07-2017; R. Sánchez-Villegas et al., 442RSV17; UPOS-104274.

Citamos estas poblaciones por tratarse de una especie muy poco común en la sierra de Gredos, si bien ha sido ya señalada para las provincias de Ávila (Soria et al., 1987) y Salamanca (Elías et al., 2001).

Lophoziopsis excisa (Dicks.) Konstant. et Vilnet

Ávila. Macizo occidental de Gredos, Solana de Ávila, central del Chorro, 40¹8'21.20"N / 540'22.10'W, $1444 \mathrm{~m}$, base de pared con escorrentía, 19-03-2017; S. Guerra-Cárdenas et al., 102SGC17bis; UPOS-104235.

Segunda cita provincial (Elías et al., 2006) de esta hepática que es poco común en Gredos, si bien es frecuente en puntos de la mitad norte peninsular (Casas et al., 2006).

Marsupella funckii (F.Weber et D.Mohr) Dumort.

${ }^{*}$ Cáceres. Macizo central de Gredos, Tornavacas, garganta de San Martín, 40¹3'44.47'N / 5³7'30.53”'W, $2057 \mathrm{~m}$, bloque granítico, 02-09-2017; I. Jurado-Castillo et al., 105IJC17; UPOS-104388.

Especie muy común sobre los granitos de las altitudes medias de Gredos (Luceño et al., 2016a) que no había sido colectada en la región extremeña.

\section{*Mesoptychia heterocolpos (Thed. ex Hartm.) L.Söderstr. et Váňa \\ *Salamanca. Macizo occidental de Gredos,}

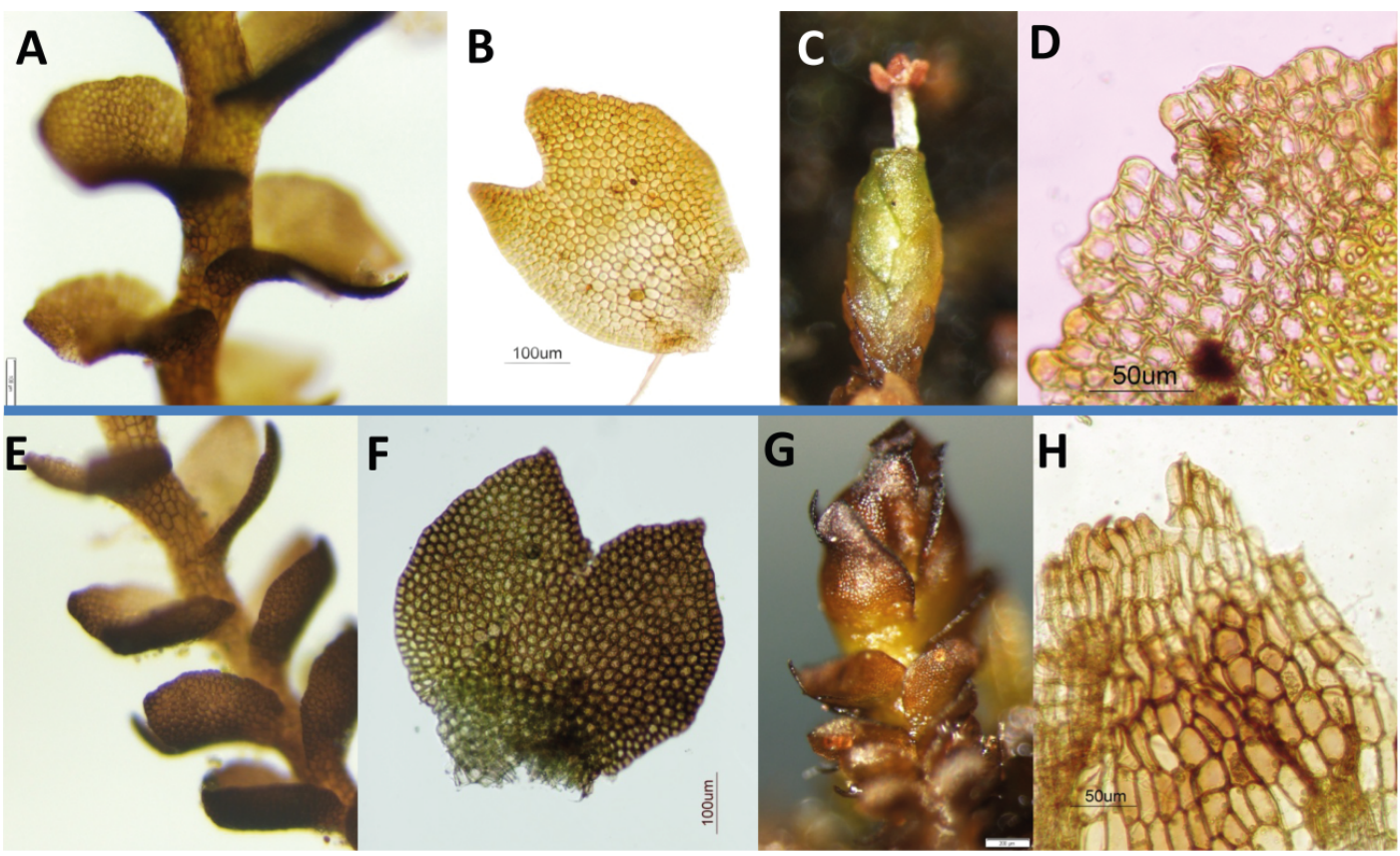

Figura 1. Gymnomitrion alpinum (A-D). A: filidios envainadores; B: filidio; C: inflorescencia femenina; D: ápice del perianto. Marsupella funckii (E-H). E: filidios no envainadores; F: filidio; G: inflorescencia femenina; H: ápice del perianto. Figure 1. Gymnomitrion alpinum (A-D). A: seathing leaves; B: leaves; $\boldsymbol{C}$ : female inflorescence; $\boldsymbol{D}$ : periant apex. Marsupella funckii (E-H). E: not seathing leaves; $\boldsymbol{F}$ : leaves; $\boldsymbol{G}$ : female inflorescence; $\boldsymbol{H}$ : periant apex. 
Candelario, circo de Hoya Mayor, 40²0’04.05” N / 543'13.85' $\mathrm{W}, 1986 \mathrm{~m}$, oquedades profundas de paredes graníticas tras una cascada con exposición norte, 05-082017; R. Sánchez-Villegas et al., 519RSV17; UPOS104286.

La primera referencia carpetana de este taxon ha sido publicada recientemente (Luceño et al., 2017). La localidad que aportamos ahora se encuentra bastante alejada de la que citamos anteriormente y suma una especie más al catálogo de hepáticas de la provincia de Salamanca.

\section{Pseudolophozia sudetica (Nees ex Huebener) Konstant. \& Vilnet. \\ *Cáceres. Macizo central de Gredos, Tornavacas,} garganta de San Martín, 40¹3'47.07'N / 5³7'32.89'W, 1975 m, bloques graníticos, 02-09-2017; I. JuradoCastillo et al., 91IJC17; UPOS-104337. Idem, circo de la Angostura, 40¹2'35.59' $\mathrm{N}$ / 5⒊'13.44' 'W, $2061 \mathrm{~m}$, roquedos graníticos con exposición norte, 23-09-2017; R. Sánchez-Villegas et al., 694RSV17; UPOS-104347.

Especie muy común en los pisos oro y crioromediterráneo de toda la sierra (Elías et al., 2006) que no se había hallado hasta la fecha en la parte extremeña de Gredos.

\section{Solenostoma obovatum (Nees) C. Massal.}

*Cáceres. Macizo central de Gredos, Tornavacas, circo de la Angostura, 40¹2'35.22”N / 5³7'09.89'W, 2059 m, paredes con escorrentía, 23-09-2017; R. Sánchez-Villegas et al., 697RSV17; UPOS-104268.

Planta bastante frecuente sobre granitos húmedos de las partes elevadas de toda la sierra (Luceño et al., 2017) que no había sido citada hasta ahora de Extremadura.

\section{MUSGOS}

\section{Andreaea alpestris (Thed.) Schimp.}

*Cáceres. Macizo central de Gredos, Tornavacas, circo de la Angostura, 40¹2'29.52'” / 5³7'18.60'W, $2175 \mathrm{~m}$, roquedos y fisuras graníticas orientadas al norte, 23-09-2017; R. Sánchez-Villegas et al., 676RSV17; UPOS-104288.

Al igual que en el tramo abulense de Gredos, esta especie se comporta en el crioromediterráneo extremeño como vicariante altitudinal de su pariente próximo $A$. rupestris Hedw.

\section{Andreaea frigida Huebener}

${ }^{*}$ Cáceres. Macizo central de Gredos, Tornavacas, garganta de San Martín, 40¹3'44.47'N / 5³7'30.53’W, $2057 \mathrm{~m}$, bloque granítico con escorrentía temporal, 02-09-2017; I. Jurado-Castillo et al., 106IJC17; UPOS104302. Idem, circo de la Angostura, 40¹2'29.52'N / 537'18.60' $\mathrm{W}, 2175 \mathrm{~m}$, roquedos y fisuras graníticas temporalmente húmedas y orientadas al norte, 23-092017; R. Sánchez-Villegas et al., 682RSV17; UPOS104284.
Especie frecuente en los granitos con escorrentía temporal de los pisos oro y crioromediterráneo de toda la sierra que, sin embargo, no había sido colectada de la parte extremeña.

Andreaea heinemannii Hampe \& Müll. Hal.

${ }^{*}$ Cáceres. Sierra de Gata, Valverde del Fresno, Los Llanos, 40 ${ }^{\circ} 15^{\prime} 21.61$ 'N / 6 ${ }^{\circ} 51^{\prime} 11.05$ 'W, $1100 \mathrm{~m}$, grietas en bloques graníticos meteorizados, 21-05-2016; M. Luceño et al., 225ML16; UPOS-103627. Idem, macizo central de Gredos, Tornavacas, circo de laAngostura, 40¹2'30.22'N / 5³7'15.16'W, 2149 m, bloques graníticos, 23-09-2017; R. Sánchez-Villegas et al., 692RSV17; UPOS-104280. *Salamanca. Macizo occidental de Gredos, Candelario, La Covatilla, 40²1'20.01'N / 541'13.34'W, 1960 m, bloques graníticos expuestos al norte, 05-12-2016; S. Guerra-Cárdenas et al., 756SGC17; UPOS-104144. Idem, Candelario, circo de Hoya Mayor, 40²0'09.95"N / 543'29.05' W, $1884 \mathrm{~m}$, paredes graníticas frescas y umbrías, 15-04-2017; S. Guerra-Cárdenas et al., 300SGC17; UPOS-104258.

Aunque Flora Briofítica Ibérica (Cross \& Sérgio, 2007) ha admitido dos subespecies para A. heinemannii [subsps. heinemannii y crassifolia (Luisier) Sérgio], nosotros creemos que ambos táxones deben ser considerados con el rango de especie (Dirkse \& Losada-Lima, 2010; véase también Luceño et al., 2016b). Nuestra cita de ahora es una novedad para Extremadura y Salamanca. A. heinemannii es una especie bastante frecuente en Gredos, aunque difícil de detectar debido al pequeño tamaño de sus cojinetes (Luceño et al., 2017).

\section{Andreaea rupestris Hedw.}

${ }^{*}$ Cáceres. Macizo central de Gredos, Tornavacas, garganta de San Martín, 40¹3'48.85”N / 5³8'28.02”W, $1761 \mathrm{~m}$, roquedos con exposición norte, 23-09-2017; R. Sánchez-Villegas et al., 621RSV17; UPOS-104282.

Especie frecuente en los niveles medios de toda la sierra que, sorprendentemente, no se había colectado en Cáceres.

\section{Bryum muehlenbeckii Bruch \& Schimp.}

*Cáceres. Macizo central de Gredos, Tornavacas, garganta de San Martín, 40¹3'38.17'N / 5³6'57.52'W, 2215 m, junto arroyo, 02-09-2017; I. Jurado-Castillo et al., 146IJC17; UPOS-104307. Idem, circo de la Angostura, 40¹2'43.32'N / 5³7'20.77''W, $1984 \mathrm{~m}$, roquedos, 2309-2017; R. Sánchez-Villegas et al., 645RSV17; UPOS104298.

Muy frecuente y abundante en los circos de San Martín y La Angostura. Es una especie muy común en las zonas adyacentes del Gredos abulense y salmantino.

*Diphyscium foliosum (L. ex Hedw.) D. Mohr Ávila. Macizo occidental de Gredos, Solana de Ávila, 
laguna del Chorrito, 40²0'32.27'N / 540'21.15”W, 2029 $\mathrm{m}$, taludes húmedos en arroyo, 31-08-2016; S. GuerraCárdenas et al., 440SGC16; UPOS-103688. Idem, Hoyo Malillo, 40¹7'50.03'N / 541'33.10'W, $1823 \mathrm{~m}$, taludes junto a arroyo, 01-10-2016; S. Guerra-Cárdenas et al., 591SGC16; UPOS-104018.

Especie no conocida del macizo occidental de Gredos, donde parece no ser una rareza a pesar de que no resulta fácil detectarla. Del macizo central fue citada por Lloret et al. (1997).

\section{Grimmia anomala Hampe ex Schimp.}

*Cáceres. Macizo central de Gredos, Tornavacas, garganta de San Martín, 40¹3'40.90”N / 5³7'25.73”W, $2130 \mathrm{~m}$, sobre bloque granítico, 02-09-2017; I. JuradoCastillo et al., 117IJC17; UPOS-104310.

Relevante novedad para Extremadura de una especie poco frecuente en la cordillera (Luceño et al., 2016a) y rara en el conjunto de la Península Ibérica (Muñoz et al., 2015).

\section{Grimmia caespiticia (Brid.) Jur.}

*Cáceres. Macizo central de Gredos, Tornavacas, garganta de San Martín, 40¹3'28.09'” / 5³7'00.25”W, 2277 m, bloque granítico, 02-09-2017; I. JuradoCastillo et al., 130IJC17; UPOS-104311. Idem, circo de la Angostura, 40¹2'45.34'N / 5³7'20.77'W, 1960 $\mathrm{m}$, roquedos, 23-09-2017; R. Sánchez-Villegas et al., 639RSV17; UPOS-104275.

Aunque poco citada de Gredos hasta fechas recientes (Muñoz et al., 2015), esta especie es de presencia constante en los granitos con escorrentía temporal de las zonas elevadas de toda la sierra, tanto en las provincias de Salamanca y Ávila, como en Cáceres. Nuestras colectas de ahora son las primeras llevadas a cabo en la región extremeña.

\section{*Grimmia incurva Schwägr.}

Ávila. Macizo central de Gredos, Bohoyo, circo de la Cocinilla, 40¹5'22.75'N / 5²4'56.44'W, 2199 m, roquedos graníticos, 04-07-2017; R. Sánchez-Villegas et al., 217RSV17; UPOS-104339.

Interesante novedad para el macizo central, ya que es una especie muy rara en la Península. Hasta el momento se conocía exclusivamente del Pirineo central, de la sierra de Guadarrama y del macizo occidental de Gredos (Muñoz et al., 2015; Luceño et al., 2016a).

\footnotetext{
Hygrohypnum ochraceum (Turner ex Wilson) Loeske

*Cáceres. Macizo central de Gredos, Tornavacas, garganta de San Martín, 40¹3'38.17'N / 5³6'57.52'W, $2215 \mathrm{~m}$, junto arroyo, 02-09-2017; I. Jurado-Castillo et al., 134IJC17; UPOS-104312. Idem, circo de la Angostura, 40¹2'53.68' N / 5³7'26.33”'W, 1879 m, lecho de arroyo, 23-09-2017; R. Sánchez-Villegas et al., 630RSV17; UPOS-104278.
}

Esta especie es la más común del género en los niveles altos de la sierra, aunque no había sido colectada hasta el momento en el tramo extremeño.

\section{Hymenoloma crispulum (Hedw.) Ochyra}

${ }^{*}$ Cáceres. Macizo central de Gredos, Tornavacas, garganta de San Martín, 40¹3'39.61'”N / 5³7'23.32”W, 2165 m, bloque granítico, 02-09-2017; I. Jurado-Castillo et al., 127IJC17; UPOS-104313. Idem, circo de la Angostura, 40¹2'35.51' $\mathrm{N} / 5^{\circ} 37^{\prime} 16.77^{\prime \prime} \mathrm{W}, 2059 \mathrm{~m}$, repisas y paredes graníticas, 23-09-2017; R. SánchezVillegas et al., 663RSV17; UPOS-104341.

Musgo muy común en los niveles altos de los macizos central y occidental de Gredos (Luceño et al., 2016a), si bien no había sido colectada hasta el momento del tramo extremeño.

Kiaeria blyttii (Bruch \& Schimp.) Broth.

*Salamanca. Macizo occidental de Gredos, Candelario, circo Hoya Mayor, 40²0'03.00”'N / 543'16.26'W, $1950 \mathrm{~m}$, canchales, 15-04-2017; M. Luceño et al., 212ML17; UPOS-104393.

Novedad para Salamanca. Es una especie rara en el Sistema Central y en el conjunto peninsular (Luceño et al., 2017).

\section{Kiaeria starkei (F. Weber \& D. Mohr) I. Hagen}

${ }^{*}$ Cáceres. Macizo central de Gredos, Tornavacas, garganta de San Martín, 40¹3'28.09”N / 5³7’00.25”W, 2277 m, bloque granítico, 02-09-2017; I. Jurado-Castillo et al., 129IJC17; UPOS-104314. Idem, circo de la Angostura, 40¹2'35.59' $\mathrm{N} / 5^{\circ} 37^{\prime} 13.44^{\prime \prime} \mathrm{W}, 2061 \mathrm{~m}$, roquedos graníticos con exposición norte, 23-09-2017; R. Sánchez-Villegas et al., 693RSV17; UPOS-104271.

Novedad para la brioflora de Extremadura. K. starkei es muy abundante en todos los tramos elevados de la sierra (Luceño et al., 2016a).

\section{Lescuraea incurvata (Hedw.) E. Lawton}

${ }^{*}$ Cáceres. Macizo central de Gredos, Tornavacas, garganta de San Martín, 40¹3'38.17'N / 5³6'57.52”W, $2215 \mathrm{~m}$, junto arroyo, 02-09-2017; I. Jurado-Castillo et al., 149IJC17; UPOS-104315. Idem, circo de la Angostura, 40¹2'28.70”'N / 5³7'20.04”W, $2180 \mathrm{~m}$, recovecos graníticos, 23-09-2017; R. Sánchez-Villegas et al., 665RSV17; UPOS-104342.

Novedad para la comunidad de Extremadura. Se trata de una especie bóreo-alpina bastante común en los niveles altos de Gredos (Luceño et al., 2016a).

\section{Lescuraea patens Lindb.}

${ }^{*}$ Cáceres. Macizo central de Gredos, Tornavacas, garganta de San Martín, 40¹3'48.64'” / 5³7'35.71'”W, 1938 m, canchal, 02-09-2017; I. Jurado-Castillo et al., 77IJC17; UPOS-104316. Idem, circo de la Angostura, 4012'40.65”N / 537'17.45'W, $2012 \mathrm{~m}$, roquedos y paredes graníticas, 23-09-2017; R. Sánchez-Villegas et al., 656RSV17; UPOS-104277. 
Muy común en fisuras y repisas umbrosas del piso crioromediterráneo de toda la sierra (Luceño et al., 2016a), aunque no había sido colectada en la región extremeña.

\section{Lescuraea saxicola (Schimp.) Molendo}

*Cáceres. Macizo central de Gredos, Tornavacas, garganta de San Martín, 4013'38.61'N / 5⒊'23.32”'W, $2165 \mathrm{~m}$, bloque granítico, 02-09-2017; I. Jurado-Castillo et al., 120IJC17; UPOS-104321. Idem, circo de la Angostura, 40¹2'29.33” N / 5037'19.54' $\mathrm{W}, 2168 \mathrm{~m}$, recovecos graníticos, 23-09-2017; R. Sánchez-Villegas et al., 669RSV17bis; UPOS-104273.

Más rara que sus congéneres en los circos extremeños, fue citada recientemente de la sierra de Gredos (Luceño et al., 2017), aunque nuestro hallazgo de ahora supone una novedad para Extremadura.

\section{Pohlia wahlenbergii var. glacialis (Brid.) E.F.} Warb.

*Salamanca. Macizo occidental de Gredos, Candelario, sendero del Travieso a Hoya Moros, $40^{\circ} 19^{\prime} 04.44^{\prime \prime} \mathrm{N} / 5^{\circ} 43^{\prime} 59.48^{\prime \prime} \mathrm{W}, 2103 \mathrm{~m}$, granitos en recovecos recorridos por arroyo, 01-09-2016; S. GuerraCárdenas et al., 493SGC16; UPOS-104348.

Este taxon ha sido citado recientemente del Sistema Central (Luceño et al., 2017). La localidad que aportamos ahora es la primera salmantina.

\section{Polytrichastrum alpinum (Hedw.) G.L. Sm.}

*Cáceres. Macizo central de Gredos, Tornavacas, garganta de San Martín, 4013'45.50”N / 5⒊'27.49”'W, 2057 m, bloque granítico, 02-09-2017; I. Jurado-Castillo et al., 111lJC17; UPOS-104323. Idem, circo de la Angostura, 40 $12^{\prime} 29.96^{\prime \prime} \mathrm{N} / 5^{\circ} 37^{\prime} 16.76^{\prime \prime} \mathrm{W}, 2171 \mathrm{~m}$, paredes graníticas, 23-09-2017; R. Sánchez-Villegas et al., 686RSV17; UPOS-104279.

A pesar de su frecuencia y abundancia en las partes altas de toda la sierra de Gredos, este musgo no había sido citado aún de Extremadura.

\section{Pseudotaxiphyllum elegans (Brid.) Z. Iwats.}

${ }^{*}$ Cáceres. Macizo central de Gredos, Tornavacas, garganta de San Martín, 40¹3'38.17'N / 5³6'57.52'W, $2215 \mathrm{~m}$, junto a arroyo, 02-09-2017; I. Jurado-Castillo et al., 147IJC17; UPOS-104324. Idem, circo de la Angostura, 40¹2'35.22”'N / 5³7'09.89'W, 2059 m, paredes con escorrentías, 23-09-2017; R. SánchezVillegas et al., 705RSV17; UPOS-104292.

Novedad para Extremadura. Se trata de una especie bastante común en los pisos supra, oro y, especialmente, crioromediterráneo.

\section{Racomitrium macounii subsp. alpinum (E. Lawton) Frisvoll \\ *Cáceres. Macizo central de Gredos, Tornavacas,} garganta de San Martín, 40¹3’44.05”N / 5³7’20.61 ’W,
$2078 \mathrm{~m}$, base de bloque granítico, 02-09-2017; I. JuradoCastillo et al., 114IJC17; UPOS-104325. Idem, circo de la Angostura, 4012'40.65” N / 53'17.45”'W, 2012 m, roquedos y paredes graníticas, 23-09-2017; R. SánchezVillegas et al., 655RSV17bis; UPOS-104299.

Taxon común en los roquedos con escorrentía temporal de la zona de cumbres de toda la sierra, aunque no colectado previamente en Extremadura.

Racomitrium macounii Kindb. subsp. macounii

*Cáceres. Macizo central de Gredos, Tornavacas, garganta de San Martín, 40¹3'38.17'N / 5'36'57.52'”, $2215 \mathrm{~m}$, junto arroyo, 02-09-2017; I. Jurado-Castillo et al., 153IJC17; UPOS-104328. Idem, circo de la Angostura, $40^{\circ} 12^{\prime} 40.65^{\prime \prime} \mathrm{N} / 5^{\circ} 37^{\prime} 17.45^{\prime \prime} \mathrm{W}, 2012 \mathrm{~m}$, roquedos y paredes graníticas, 23-09-2017; R. Sánchez-Villegas et al., 652RSV17; UPOS-104392.

Más frecuente aún en Gredos que la subespecie alpinum, con la que convive frecuentemente. No había sido citada hasta el momento de la región extremeña.

Racomitrium sudeticum (Funck) Bruch \& Schimp.

*Cáceres. Macizo central de Gredos, Tornavacas, garganta de San Martín, 40¹3'39.61'N / 5³7'23.32'W, $2165 \mathrm{~m}$, bloque granítico, 02-09-2017; I. Jurado-Castillo et al., 125IJC17; UPOS-104329.

Primera cita para Cáceres y la comunidad de Extremadura.

\section{Sanionia uncinata (Hedw.) Loeske}

*Cáceres. Macizo central de Gredos, Tornavacas, circo de la Angostura, $40^{\circ} 12^{\prime} 35.22^{\prime \prime} \mathrm{N} / 5^{\circ} 37^{\prime} 09.89^{\prime \prime} \mathrm{W}$, 2059 m, paredes con escorrentías, 23-09-2017; R. Sánchez-Villegas et al., 708RSV17; UPOS-104293. $40^{\circ} 13^{\prime} 38.17^{\prime \prime} \mathrm{N} / 5^{\circ} 36^{\prime} 57.52^{\prime \prime} \mathrm{W}, 2215 \mathrm{~m}$, junto arroyo, 02-09-2017; I. Jurado-Castillo et al., 156IJC17; UPOS104335.

Relativamente común en las turberas y humedales de la sierra, su presencia en el tramo extremeño no se había constatado.

Sarmentypnum exannulatum (Schimp.) Hedenäs

*Cáceres. Macizo central de Gredos, Tornavacas, garganta de San Martín, 40¹3'55.79"N / 5'38'24.23'W, $1681 \mathrm{~m}$, turbera, 23-09-2017; R. Sánchez-Villegas et al., 601RSV17; UPOS-104390. Idem, lagunillas del circo de la Angostura, 40 $12^{\prime} 41^{\prime \prime} \mathrm{N} / 5^{\circ} 37^{\prime} 07.00^{\prime \prime} \mathrm{W}, 1970$ m, turberas, 23-09-2017; R. Sánchez-Villegas et al., 712RSV17; UPOS-104309.

Musgo muy común en aguazales y turberas de toda la sierra, aunque no había sido colectado en Extremadura.

\section{Schistidium agassizii Sull. \& Lesq.}

*Cáceres. Macizo central de Gredos, Tornavacas, circo de la Angostura, 4012'53.68' $\mathrm{N} / 5^{\circ} 37^{\prime} 26.33^{\prime \prime} \mathrm{W}$, 1928 m, lecho de arroyo, 23-09-2017; R. SánchezVillegas et al., 635RSV17; UPOS-104295. 
Especie poco frecuente en la Península, de donde se conocía exclusivamente del Pirineo Central, Sierra Nevada y el tramo abulense del macizo central de Gredos (Suárez \& Muñoz, 2015).

*Sciuro-hypnum reflexum (Starke) Ignatov \& Huttunen

Salamanca. Macizo central de Gredos, Solana de Ávila, Peña Negra, 40²0'55.26”N / 540'24.69”'W, 2085 $\mathrm{m}$, suelo en base de bloque granítico, 31-08-2016; S. Guerra-Cárdenas et al., 418SGC16bis; UPOS -103787.

Relevante novedad para el Sistema Central. Este musgo se conocía exclusivamente de Sierra Nevada, Pirineos y puntos aislados de la Cordillera Cantábrica (Orgaz, 2012).

\section{Straminergon stramineum (Dicks. ex Brid.) Hedenäs \\ ${ }^{*}$ Cáceres. Macizo central de Gredos, Tornavacas,} circo de la Angostura, 4012'41" N / 5 ${ }^{\circ} 37^{\prime} 7^{\prime \prime} \mathrm{W}, 1970$ m, turbera, 23-09-2017; R. Sánchez-Villegas et al., 713RSV17; UPOS-104276.

Musgo común en las turberas gredenses que aún no se había colectado en la región extremeña.

\section{Syntrichia norvegica F. Weber}

${ }^{*}$ Cáceres. Macizo central de Gredos, Tornavacas, garganta de San Martín, 40¹3'38.17'N / 5³6'57.52”W, $2215 \mathrm{~m}$, junto arroyo, 02-09-2017; I. Jurado-Castillo et al., 163IJC17; UPOS-104330.

Relevante novedad para Extremadura; esta especie bóreo-alpina ha sido recientemente citada del Sistema Central (Luceño et al., 2017).

Thuidium tamariscinum (Hedw.) Schimp.

*Ávila. Macizo occidental de Gredos, Solana de Ávila , central del Chorro, 40¹8'21.20”N / 540'22.10”W, $1430 \mathrm{~m}$, base de bloque granítico rezumante muy umbrío junto a brezos, 19-03-2017; S. Guerra-Cárdenas et al., 79SGC17; UPOS-104204.

No conocemos citas precisas de este musgo en la sierra de Gredos, si bien su presencia en Cáceres y Salamanca es recogida en Brugués \& Ruiz (en prensa).

\section{* Tortula hoppeana (Schultz) Ochyra}

Ávila. Macizo central de Gredos, Zapardiel de la Ribera, Circo del Güetre, 40¹5'31.70'N / 5¹7'57.00'W, $2350 \mathrm{~m}$, torrentes graníticos quionófilos, 21-07-2017; R. Sánchez-Villegas et al., 425RSV17; UPOS-104389. Cáceres. Macizo central de Gredos, Tornavacas, garganta de San Martín, 40¹3'48.64"N / 5³7'35.71 'W, 1938 m, canchal, 02-09-2017; I. Jurado-Castillo et al., 84IJC17; UPOS-104331. Idem, circo de la Angostura, 40 12'29.33”' / 5³7'19.54”'W, $2168 \mathrm{~m}$, recovecos graníticos, 23-09-2017; R. Sánchez-Villegas et al., 667RSV17bis; UPOS-104297.

Relevante novedad para el Sistema Central y la Comunidad Autónoma de Extremadura. La presencia de esta especie bóreo-alpina en la Península se reduce a las montañas del norte y a Sierra Nevada (Cano, 2006).

\section{Agradecimientos}

Agradecemos a Marta Infante (Vitoria) la identificación de la muestra de Douinia ovata.

\section{Referencias}

Brugués, M. \& Ruiz, E. -en prensa- Thuidium Bruch \& Schimp. En: Guerra, J., M. J. Cano \& R. M. Ros (eds.), Flora Briofítica Ibérica.

Cano, M. J. (2006). Tortula. En: Guerra, J., M. J. Cano \& R. M. Ros (eds.), Flora Briofítica Ibérica. Volumen III, pp. 146-176. Universidad de Murcia - Sociedad Española de Briología. Murcia.

Casares Gil, A. \& Beltrán Bigorra, F. (1912). Flora briológica de la Sierra de Guadarrama. Trab. Mus. Nac. Ci. Na. Ser. Bot., 1, 1-50.

Casas, C., Brugués, M., Cros, R. M. \& Sérgio, S. (1985). Bryophyte Cartography: Iberian Peninsula, Balearic and Canary Islands, Azores and Madeira. Fascicle I (1-50). Institut d'Estudis Catalans. Barcelona.

Casas, C., Brugués, M. \& Cros, R.M. (1988). La brioflora de la Sierra de Gata. Orsis, 3, 27-40.

Casas, C., Brugués, M., Cros, R. M. \& Sérgio, C. (2006). Handbook of mosses of the Iberian Peninsula and the Balearic Islands. Institut d'Estudis Catalans. Barcelona.

Cros, R.M. \& Sergio, C. (2007). Andreaea. En Brugués, M., Cros, R.M. \& Guerra, J. (eds.). Flora Briofítica Ibérica. Volumen 1. Universidad de Murcia - Sociedad Española de Briología, pp. 81-98. Murcia.

Elías, M. J. (1988). Fragmenta Chorologica Occidentalia, Bryophyta, 1458-1493. Anales Jard. Bot. Madrid, 45, 303-307.

Elías, M.J., Sánchez, J.A. \& García, J.M. (2001). Hepáticas y antocerotas (Marchantiopsida y Anthocerotopsida) de Salamanca (España). Bot. Complutensis, 25, 207-212.

Elías, M.J., Albertos, B., Brugués, M., Calabrese, G., Cano, M.J., Estébanez, B., Gallego, M.T., Garilleti, R., Guerra, J., Heras, P., Infante, M., Lara, F., Martín, M.A., Mazimpaka, V., Medina, R., Muñoz, J., Pokorny, L., Puche, F. \& Sánchez, J.A. (2006). Aportaciones al conocimiento de la flora briológica española. Nótula XV: musgos, antocerotas y hepáticas de la Sierra de Gredos (Ávila). Bol. Soc. Esp. Briol. 28: 25-31.

Dirkse, G.M. \& Losada-Lima, A. (2010). Andreaea Hedw. in the Canary Islands. J. Bryol., 32, 51-55.

Guerra, J., Brugués, M., Cano, M.J. \& Cros, R.M., eds. (2010). Flora Briofítica Ibérica. Volumen IV. Universidad de Murcia - Sociedad Española de Briología. Murcia.

Hespanhol, H., Séneca, A. \& Sérgio, C. (2010). Bryophytes from exposed rock outcrops in the north and centre of Portugal: distribution and conservation. Bol. Soc. Esp. Briol., 34/35, 19-35.

Lloret, F., Cros, R. M., Brugués, M. \& Granzow De La Cerda, Í. (1997). Aspectos biogeográficos y 
corológicos de los briófitos de la Sierra de Gredos (España). Cryptogamie, Bryol. Lichenol., 18, 151-164.

Luceño, M., Cerrejón, C., Guerra-Cárdenas, S., MárquezCorro, J.I., Pineda-Labella, V., Infante, M. \& Muñoz, J. (2016a). Novedades para la brioflora de la Sierra de Gredos (Sistema Central, España). Bol. Soc. Esp. Briol., 46/47, 43-68.

Luceño, M., Vargas, P. \& García, B. (2016b). Guía de campo del Sistema Central. Raíces, Madrid.

Luceño, M., Cerrejón, C., Guerra-Cárdenas, S., Márquez-Corro, J.I., Pineda-Labella, V., Martín-Bravo, S., Infante, M. \& Muñoz, J. (2017). A contribution to the knowledge of bryophytes from Sierra de Gredos (central Spain) including a reevaluation of their national conservation status. Cryptogam. Bryol., 38(3), 281-302.

Muñoz, J., Cezón, K., Hespanhol, H. \& Quandt, D. (2015). Grimmia. En: Brugués, M. \& J. Guerra (eds.), Flora Briofítica Ibérica. Volumen II, pp. 210261. Universidad de Murcia - Sociedad Española de Briología. Murcia.

Orgaz, J. D. (2012). Brachythecium. En: Guerra, J. \& R. M. Cros, Coords., Flora Briofítica Ibérica (Fascículos). Universidad de Murcia - Sociedad Española de Briología. Murcia.

Paton, J. A. (1999). The liverwort flora of the British Isles. Harley Books, England.

Ros, R.M., Mazimpaka, V., Abou-Salama, U., Aleffi, M., Blockeel, T.L., Brugués, M., Cros, R.M., Dia, M.G., Dirkse, G.M., Draper, I., El-Saadawi, W., Erdag, A.,
Ganeva, A., Gabriel, R., Gonzalez-Mancebo, J.M., Granger, C., Herrnstadt, I., Hugonnot, V., Khalil, K., Kurschner, H., Losada-Lima, A., Luis, L., Mifsud, S., Privitera, M., Puglisi, M., Sabovljevic, M., Sergio, C., Shabbara, H.M., Sim-Sim, M., Sotiaux, A., Tacchi, R., Vanderpoorten, A. \& Werner, O. (2013). Mosses of the Mediterranean, an annotated checklist. Cryptogamie, Bryologie 34: 99-283.

Söderström, L., Hagborg, A., Konrat, M.V., BartholomewBegan, S., Bell, D., Briscoe, L., Brown, E., Cargill, D.C., Costa, D.P.D., Crandall-Stotler, B.J., Cooper, E.D., Dauphin, G., Engel, J., Feldberg, K., Glenny, D., Gradstein, S.R., He, X., Hentschel, J., IlkiuBorges, A.L., Katagiri, T., Konstantinova, N.A., Larraín, J., Long, D., Nebel, M., Pócs, T., Puche, F., Reiner-Drehwald, E., Renner, M., Sass-Gyarmati, A., Schäferverwimp, A., Segarra-Moragues, J.G., Stotler, R.E., Sukkharak, P., Thiers, B., Uribe, J., Vána, J., Wigginton, M., Zhang, L. \& Zhu, R.-L. (2016). World checklist of hornworts and liverworts. Phytokeys, 59, 1-828.

Soria, A., Mazimpaka, V., Riestra, P. \& Ron, M.E. (1987). Aportaciones al conocimiento de la brioflora del Puerto del Pico, Sierra de Gredos (Ávila). Actas del IV Simposio Nacional de Botánica Criptogámica. Universidad de Granada, Granada, 619-628.

Suárez, G. M. \& Muñoz, J. -(2015). Schistidium. En: Brugués, M. \& J. Guerra (eds.), Flora Briofítica Ibérica. Volumen II, pp. 290-325. Universidad de Murcia Sociedad Española de Briología. Murcia. 